\title{
Los impactos de la nueva política de salario mínimo. El caso de Jalisco y Baja California
}

\author{
JOSÉ MARÍA PARRA RUIZ ${ }^{1}$ \\ EMILIA GÁMEZ FRÍAS ${ }^{2}$
}

\section{Resumen}

En 2019 se impulsa una nueva política del salario mínimo que configura dos regiones salariales: Zona Libre de la Frontera Norte de México y Resto del país. Para la primera región se autoriza un incremento del $100 \%$, y para la segunda del $16.2 \%$. Nuestro objetivo es analizar el impacto que produce, en 2019, el incremento diferencial del salario mínimo en la generación de nuevos empleos de la fuerza de trabajo asalariada (población objetivo del salario mínimo) de Jalisco (entidad emblemática de la región resto de México) y de Baja California (entidad de la región de la Zona Libre de la Frontera Norte de México) contrastándolo con el promedio nacional en tres niveles: estructura ocupacional, sector y actividad económica, y por tamaño de establecimiento, así como por sexo. La evidencia estadística la procesamos de la encuesta nacional de la ocupación y el empleo (INEGI).

Palabras clave: política de salario mínimo, ocupación, fuerza de trabajo asalariada, empleo asalariado

Clasificación JEL: R23

Fecha de recepción: 22 de enero de 2020. Fecha de aceptación: 17 de agosto de 2020.

1. Profesor-investigador de tiempo completo con 30 años de antigüedad. Departamento de Estudios Regionales-INESER, Centro Universitario de Ciencias Económico Administrativas (CUCEA), Universidad de Guadalajara. Responsable del CA118: Mercados de trabajo y Desarrollo Territorial; Perfil PRODEP. Orcid ID: https://orcid.org/0000-0002-6395-9839. Dirección electrónica: jparra@ cucea.udg.mx.

2. Profesora-docente de tiempo completo con 31 años de antigüedad. Miembro del CA118: Mercados de trabajo y Desarrollo Territorial; Perfil PRODEP. Departamento de Estudios Regionales-INESER, Centro Universitario de Ciencias Económico Administrativas (CUCEA), Universidad de Guadalajara. Dirección electrónica: emigamez@cucea.udg.mx. 


\title{
THE IMPACTS OF THE NEW MINIMUM WAGE POLICY. THE CASE OF JALISCO AND BAJA CALIFORNIA
}

\begin{abstract}
In 2019, a new minimum wage policy is promoted that configures two wage regions: the free zone of the northern border of Mexico and the rest of the country. For the first region an increase of 100 percent is authorized and for the second of 16.2 percent. Our objective is to analyze the impact that, in 2019, the differential increase of the minimum wage in the generation of new jobs of the salaried labor force (target population of the minimum wage) of Jalisco (emblematic entity of the rest of Mexico region) and of Baja California (entity of the region of the free zone of the northern border of Mexico) contrasting it with the national average in three levels: occupational structure, sector and economic activity and by size of establishment, as well as by sex. The statistical evidence is processed by the national survey of occupation and employment (INEGI).

Keywords: minimum wage policy, occupation, salaried workforce, salaried employment.

JEL classification: $\mathrm{R} 23$
\end{abstract}

\section{Introducción}

En julio de 2018, México experimenta una tercera alternancia política en la administración federal encabezada por Andrés Manuel López Obrador. Como presidente en funciones, formula e impulsa un conjunto de medidas y programas tendientes a promover el desarrollo socioeconómico de México. Entre las nuevas políticas cabe destacar, a efectos del presente trabajo, la correspondiente a la nueva política de salario mínimo. Esta nueva política salarial formulada en el seno de la Comisión Nacional de Salarios Mínimos impulsa, a partir de enero del presente año, un aumento salarial diferencial en el territorio nacional. Este aumento tiende a configurar dos regiones salariales: Zona Libre de la Frontera Norte de México y Resto del País. Para el caso del primer territorio, conformado por 43 municipios de seis entidades (Baja California, Sonora, Coahuila, Chihuahua, Nuevo León y Tamaulipas), se impone un aumento al salario mínimo del $100 \%$, en tanto para la región Resto del País conformada, por 2,414 municipios de 30 entidades y 16 alcaldías de la ciudad de México, se otorga un incremento del $16.2 \%$ al salario mínimo nominal. Cabe señalar que dichos incrementos superan las previsiones de la inflación para este 2019, con el fin de tratar de resarcir el poder adquisitivo de los trabajadores asalariados. Esta decisión contraviene lo realizado desde hace 35 años por parte de los sucesivos gobiernos, si se considera que un incremento importante al salario mínimo trae como consecuencia pérdida sustancial de empleo, toda vez que las empresas pudieran no estar de acuerdo en cubrirlo, y también consideraban que dicho incremento produce un aumento en la 
tasa de inflación si, a la par, no se incrementa en la misma proporción la productividad laboral. Asimismo, expresaban que dichos incrementos pudieran ocasionar un "efecto faro", en el que todos los salarios de la economía se ajustaran en la misma proporción que el mínimo.

De acuerdo con lo anterior, el objetivo del presente trabajo es ofrecer evidencia estadística sobre el impacto que produce el incremento diferencial del salario mínimo en el volumen de empleo que registra la fuerza de trabajo asalariada, durante el primer semestre de 2019, en las dos regiones salariales en que se divide México. Para ello analizamos la situación que presentan, por una parte, Baja California, entidad de la región zona libre de la frontera Norte, (única donde todos sus municipios forman parte de dicha región); por otra, Jalisco como entidad que representa, de manera importante, a la región salarial Resto del País. La situación del empleo de ambas entidades la contrastamos con la correspondiente a escala nacional y la contextualizamos en el entorno macroeconómico que prevalece, con el fin de ofrecer mejores resultados analíticos. La evidencia estadística la proporcionamos a través del procesamiento y el análisis de la encuesta nacional de la ocupación y el empleo del segundo trimestre de 2019 y 2018. La evidencia sobre la generación de empleo la analizamos, en su comportamiento general y por sexo, en cuatro niveles: PEA, estructura ocupacional, fuerza de trabajo asalariada por sector y actividad económica, así como por tamaño de establecimiento.

Para cumplir con tal propósito, el trabajo se divide en siete apartados: 1) la presente introducción; 2) consideraciones de la política y de los estudios del salario mínimo, donde, por una parte, se señalan las condiciones económicas, nacionales e internacionales, que justifican y sustentan la nueva política de salario mínimo en México con incrementos diferenciales muy superiores a la tasa de inflación; por otra, se citan los estudios relevantes que ofrecen evidencia empírica sobre los impactos positivos que produce el incremento del salario mínimo en la generación de empleo; 3) Se describe el espectro poblacional que cada una de las tres entidades territoriales presenta en general, menores y mayores de quince años y por sexo; 4) Se analiza la población económicamente activa (PEA) y sus componentes de empleados y desempleados, por sexo; 5) destacamos la relevancia que adquiere la fuerza de trabajo asalariada en la estructura ocupacional, tanto en la generación de nuevos empleos como en la participación porcentual que registra cada una de las tres entidades territoriales; 6) analizamos la generación de nuevos empleos que presenta la fuerza de trabajo asalariada en los sectores y actividades económicas, así como por tamaño de establecimiento; 7) concluimos con unas consideraciones finales que enfatizan los principales hallazgos del presente trabajo. 


\section{Consideraciones de la política y de los estudios sobre el salario mínimo}

\subsection{La política de salario mínimo}

Sobre la base de un informe especial de la Dirección Técnica dirigido al Consejo de Representantes de los Salarios Mínimos (conformado por gobierno, empresarios y trabajadores), ambas instancias, dependientes de la Comisión Nacional de Salarios Mínimos, así como también basada en la propuesta de recuperación del salario mínimo a escala nacional y en la Zona Libre de la Frontera Norte, presentada por la Secretaría del Trabajo y Previsión Social a dicho Consejo de Representantes, este confirmó la existencia de condiciones económicas que justifican la fijación del salario mínimo general y de los salarios mínimos profesionales vigentes a partir del $1^{\mathrm{o}}$ de enero de 2019. Sobre la base de dichos informe y propuesta se instrumenta una nueva política de salario mínimo.

La justificación de dicha política se sustenta en un marco de referencia nacional e internacional de lo acontecido en 2018 (crecimiento económico, productividad laboral de la economía, tasa de inflación, tipo de cambio, costo unitario de la fuerza de trabajo, condiciones laborales de la fuerza de trabajo, balanza comercial, inversión, condiciones socioeconómicas de las principales empresas manufactureras, pérdida del poder adquisitivo por parte de los trabajadores asalariados, entre otros aspectos), así como en las previsiones para 2019 de tres indicadores: crecimiento económico (entre $1.7 \%$ y $2.7 \%$ de crecimiento del PIB en México para 2019); generación de empleos formales entre 670,000 y 770,000; tasa de inflación, más menos, 3.0\% (DOF, 2018). Cabe señalar que la nueva política de salario mínimo se caracteriza por establecer dos regiones salariales: Zona Libre de la Frontera Norte ${ }^{3}$ y Resto del País: ${ }^{4}$ Para la primera región se autoriza un incremento del $100 \%$ al salario mínimo (de 88.36 a 176.72 pesos) y para la segunda un incremento del $16.2 \%$ (de 88.36 a 102.68 pesos). Dichos incrementos se establecen de la siguiente manera: para la región Zona Libre Norte el incremento del $100 \%$ se establece de manera directa; en tanto para la región resto del país se otorgó un incremento directo del 5\% (4.42 pesos), y los restantes 9.9 pesos (para sumar 102.68) se otorgaron a través del Monto Independiente de Recuperación (MIR). ${ }^{5}$

3. La Zona Libre de la Frontera Norte está configurada por 43 municipios de seis entidades federales que lindan con la frontera con Estados Unidos de América. Los municipios son Tijuana, Mexicali, Rosarito, Ensenada y Tecate en Baja California. En el estado de Sonora: San Luis Río Colorado, Puerto Peñasco, General Plutarco Elías Calles, Caborca, Altar, Sáric, Nogales, Santa Cruz, Cananea, Naco y Agua Prieta; en el estado de Chihuahua: Janos, Ascensión, Juárez, Práxedis G. Guerrero, Guadalupe, Coyame del Sotol, Ojinaga y Manuel Benavides; en el estado de Coahuila: Ocampo, Acuña, Zaragoza, Jiménez, Piedras Negras, Nava, Guerrero e Hidalgo; en el estado de Nuevo León: Anáhuac; y en el estado de Tamaulipas: Nuevo Laredo, Guerrero, Mier, Miguel Alemán, Camargo, Gustavo Díaz Ordaz, Reynosa, Río Bravo, Valle Hermoso y Matamoros.

4. La región Resto del País la conforman los restantes 2,414 municipios de 30 entidades federativas (con excepción de Baja California) y por las dieciséis alcaldías de la ciudad de México.

5. El MIR "se tipifica de la siguiente manera: 1) Es una cantidad absoluta en pesos. 2) Su objetivo es única y exclusivamente contribuir a la recuperación del poder adquisitivo del salario mínimo general. 3) No debe ser utilizado como referente para fijar incrementos de los demás salarios vigentes en el mercado 
Además de que la fuerza de trabajo asalariada de la región Frontera Libre Norte recibe un incremento del salario mínimo del $100 \%$, se añaden incentivos fiscales a los empleadores, tales como: reducción del impuesto al valor agregado (IVA) de $8 \%$, y del impuesto sobre la renta (ISR) a 20\% máximo, así como la homologación con los estados del sur de los Estados Unidos con respecto a los precios de gasolina, diésel, gas y energía eléctrica para toda la población. Todo ello en aras de incentivar el desarrollo de dicha región norteña.

En tanto, para el conjunto del territorio nacional se impulsan, con recursos públicos, treinta programas nacionales, tales como: desarrollo del istmo de Tehuantepec, mejoramiento urbano, caminos rurales, precios de garantía a productores del campo, crédito ganadero a la palabra, siembra de árboles frutales y forestales, pensiones a adultos mayores y con discapacidad y becas a estudiantes de diferentes niveles educativos entre otros. Asimismo, se impulsan diez proyectos, como construcción y rehabilitación de refinerías, creación de universidades públicas y nuevo aeropuerto internacional en la ciudad de México, entre los más importantes. Todo ello con el propósito de participar en la generación del mayor volumen de empleo y procurar ingresos a la población que, principalmente, vive en condiciones de pobreza, tanto en el campo como en la ciudad.

Cabe señalar que los incrementos al salario mínimo se dirigen solamente a la fuerza de trabajo asalariada que obtiene como ingreso un salario mínimo general por el desempeño de su trabajo, pero que, a su vez, tiende a incidir en el nuevo número de empleos con salario mínimo, así como en el monto total de ingresos que percibe dicha fuerza de trabajo.

\subsection{Los estudios del salario mínimo}

Desde 1982 hasta diciembre de 2018 las sucesivas administraciones federales argumentaron que un incremento al salario mínimo, por arriba de la tasa de inflación estimada, trae como consecuencia una pérdida sustancial de empleo y un aumento en el nivel general de precios. Esto presupone que un incremento del salario mínimo trae consecuencias negativas, como la pérdida de empleo o el aumento en el nivel general de precios. Sin embargo, la evidencia empírica que ofrecen numerosos estudios académicos, de países tanto desarrollados como no desarrollados, confirma que dichos impactos negativos no ocurren.

Frente a la pérdida del poder adquisitivo del salario mínimo estadounidense, durante la década de los ochenta del siglo pasado, algunos economistas se preguntaron

\footnotetext{
laboral (salarios contractuales, federales y de la jurisdicción local; salarios diferentes a los mínimos y a los contractuales; salarios para servidores públicos federales, estatales y municipales y demás salarios del sector informal). 4) El MIR podrá ser aplicado tanto en un procedimiento de revisión salarial como de fijación salarial previstos en el artículo 570 de la Ley Federal del Trabajo".

"El propósito del MIR es hacer posible que se recupere el poder adquisitivo de los trabajadores asalariados que perciben un salario mínimo general. El Consejo de Representantes acordó continuar aplicado el proceso de la MIR en la nueva fijación del salario mínimo" (véase Diario Oficial de la Federación, 2018).
} 
qué efectos podría tener un incremento de dicho salario en el nivel de empleo y en la distribución del ingreso. Para responder a dicha interrogante realizaron trabajo de campo en dos condados aledaños: en uno se ofreció un incremento al salario mínimo y en el otro no se ofreció incremento, y levantaron encuestas y entrevistas a trabajadores de comida rápida y de bajos ingresos, así como de administradores. Los resultados mostraron que ante un incremento del salario mínimo no se observaron efectos negativos en el empleo (Card y Krueger, 1994). Posteriormente, Dube, Lester y Reich (2010), utilizando la misma metodología de Card y Krueger, llegaron a las mismas conclusiones: nula afectación al nivel de empleo y efectos positivos en el ingreso. En Inglaterra, Stewar (2004) obtuvo los mismos resultados anteriores. En lo correspondiente a los países no desarrollados, y bajo la premisa de que los efectos pueden ser diferentes a los encontrados en países desarrollados, toda vez que cuentan con un número mayor de trabajadores no capacitados, se observó lo siguiente: En su estudio en Brasil, Neumart y Wascher (2006) concluyeron que, ante un incremento del $20 \%$ en el salario mínimo, durante el periodo de Lula, no se logró contar con evidencia de que hubiera un incremento en el ingreso en la parte baja de la distribución. En tanto, Lemos (2009), también en Brasil, no encontró efecto negativo en el nivel de empleo. Para el caso de Honduras, el estudio de Gindling y Terrel (2010) sobre veintidós cambios en el salario mínimo concluyó: por una parte, observaron un incremento en el salario medio de las medianas y grandes empresas y, a la vez, una reducción de empleo en estas; por otra, no encontraron incrementos en los salarios medios de los trabajadores de las pequeñas empresas, y también que existe transferencia de fuerza de trabajo de las grandes a las pequeñas empresas. En México, los estudios recientes son escasos. Kaplan y Pérez (2006), en el estudio sobre el impacto del salario mínimo durante el periodo 1985-2001, observaron que los cambios en el salario mínimo real tuvieron un impacto positivo en los ingresos laborales de todos los niveles. Es decir, el "efecto faro" fue favorable para otros niveles de ingreso, aunque su difusión tiende a diluirse rápidamente. En un estudio más reciente, Campos, Esquivel y Santillán (2017), sobre la homologación de zonas salariales en México, observaron en 2012:

que el aumento del salario mínimo no tuvo efectos negativos en términos laborales y que, por el contrario, produjo algunos efectos positivos (...) el aumento del salario mínimo no parece haber afectado los niveles de empleo en dicha muestra (...) De la misma forma, disminuyeron las probabilidades de que las personas que tenían un trabajo informal asalariado mantuvieran ese tipo de trabajo y aumentaron las probabilidades de que obtuvieran un trabajo formal (Campos et al., 2017).

Ante la dificultad de identificar el impacto que produce el salario mínimo en el nivel del empleo, el conjunto de los estudios mencionados nos ofrece una alternativa analítica importante a efectos del presente trabajo: establecer una comparación entre dos entidades territoriales donde una de ellas ofrezca un salario mínimo mayor a los trabajadores asalariados y la otra un salario menor a estos mismos trabajadores. Otra aportación importante que encontramos en dichos estudios es que el tema del impacto del salario mínimo en el nivel de empleo se revela importante y controvertido, por 
las consecuencias positivas y negativas que infligen a los distintos actores laborales, pero de forma específica a la población asalariada y a sus familias. Es decir, para nuestro trabajo es importante centrar el análisis en este segmento de trabajadores, fuerza de trabajo asalariada, toda vez que el salario mínimo se dirige directamente a ellos.

Sobre la base de estas dos consideraciones metodológicas, en el presente trabajo realizamos un análisis comparativo sobre el impacto que produce el salario mínimo que prevalece, desde el primero de enero de 2019, entre la fuerza de trabajo asalariada de Jalisco (entidad representante de la región salarial Resto del País) y de Baja California (única entidad del norte del país en la cual todos sus municipios forman parte de la región salarial: Zona Libre Norte).

A la consideración metodológica anterior añadimos la propuesta conceptual de De la Garza (2000) sobre la construcción social del mercado de trabajo. En dicha concepción, la principal consideración es que la oferta y la demanda de fuerza de trabajo son una construcción social que siempre se encuentra acotada por estructuras diversas (macro y micro), que limitan o posibilitan los encuentros (empleo) o desencuentros (desempleo), las cuales no siempre coinciden en el espacio y el tiempo. Entre las estructuras macroeconómicas se destacan las correspondientes a política económica, inversión, leyes y normatividad laboral, así como la política salarial, entre otras. Entre las estructuras micro se destacan: características propias del mercado de bienes y servicios en que compiten, tamaño del establecimiento, mercado al que dirigen sus productos (nacional, local o extranjero), productividad y localización territorial, entre otros aspectos. Asimismo, se considera que en la definición de la estrategia de empleo (número, perfil y salarios a pagar a la fuerza de trabajo) intervienen otros actores, como empresarios, gobierno y sindicatos.

De acuerdo con lo anterior, el concepto de la construcción social del mercado de trabajo resulta útil en razón de que nos permite considerar el impacto de la política del salario mínimo sobre el nivel de empleo de la fuerza de trabajo asalariada como una variable macroeconómica de la mayor importancia para analizar los encuentros y desencuentros que ocurren entre oferentes y demandantes de fuerza de trabajo en las dos regiones salariales de México, considerando el contexto del cúmulo de presiones de que son objeto dichos oferentes y demandantes.

\section{El espectro poblacional}

\subsection{Población total}

En 2019, el total de la población mexicana (125,782,872 habitantes) registra un crecimiento del 1\% respecto del año anterior (las cuales suman 1,195,748 personas más). Para este mismo año, por una parte, las mujeres experimentan un crecimiento del $0.9 \%$ (561, nuevas mujeres), y los hombres del 1.1\% (634,669 más). Por otra parte, las mujeres representan el $51.7 \%$, del total de la población y los hombres el $48.3 \%$ (cuadro 1). 
Por su parte, Jalisco registra el mismo porcentaje de crecimiento poblacional a escala nacional: $1 \%$ (84,561 personas más). En contraste con el registro nacional, las mujeres presentan un crecimiento del $1.2 \%$, y los hombres del $0.9 \%$. En cuanto a su participación, las mujeres registran, en ambos años, $51.2 \%$, y los hombres el restante $48.8 \%$ (cuadro 1 ).

Baja California registra un crecimiento del 1.3\% (48,440 personas más), en el total de población. Por sexo, las mujeres presentan un crecimiento negativo de $1.8 \%$ (32,551 menos), en tanto los hombres un crecimiento del 0.9, lo cual significa contar con 15,889 personas más. En cuanto a su participación en el total poblacional, las mujeres (no obstante que registran un crecimiento negativo) representan el 50.1\% (0.9 puntos porcentuales menos que en 2018); los hombres representan el 49.9\%; esto es, 0.7 puntos porcentuales más que el año anterior (cuadro 1).

\subsection{Población menor de 15 años}

Este segmento poblacional registra un crecimiento negativo en las tres entidades territoriales. A escala nacional el descenso es de -1.1\%; en Jalisco y en Baja California el descenso asciende a $-3.1 \%$ ( 2 puntos porcentuales superior al promedio nacional). Por sexo, las mujeres también registran, en las tres entidades, un retroceso en su crecimiento, de $-1.4 \%$ a escala nacional, $-2.4 \%$ en Jalisco y $-6,2 \%$ en Baja California. Por su parte, a escala nacional los hombres registran una caída de $-0.8 \%$, en Jalisco un crecimiento de $1.3 \%$, y en Baja California experimentan un estancamiento en su tasa de crecimiento. En términos absolutos, significa que a escala nacional se registra una pérdida total de 359,386 personas de menos de quince años. Por sexo, la pérdida asciende a 135,133 hombres y 224,253 mujeres. En Jalisco, este segmento de población registra un descenso de 10,719 personas. Por sexo, un saldo positivo de 13,698 hombres y una pérdida de 24,417 mujeres. En Baja California, una pérdida total de 27,750 personas. Por sexo, saldo positivo de 97 hombres y una pérdida de 27,847 mujeres (cuadro 1).

La participación porcentual de este segmento de población con respecto al total, en las tres entidades territoriales, experimenta, en 2019, un descenso de 0.2 puntos porcentuales en el promedio nacional, 0.4 en Jalisco y 0.9 puntos porcentuales en Baja California, como consecuencia del descenso de la población femenina, la cual se distancia del $50 \%$ de participación en cada uno de los tres territorios. En contraste, la población masculina aumenta su participación, en las tres entidades, por encima del $50 \%$ (cuadro 1).

\subsection{Población de 15 y más años}

Este segmento de población registra durante 2019, a escala territorial y por sexo, tasas de crecimiento positivas del $1.7 \%$. Se destaca el $2.8 \%$ de crecimiento de Baja California en este segmento poblacional (1.1 puntos porcentuales por arriba del promedio nacional), así como el 4.4\% de crecimiento de su población femenina (60,398 más con respecto al año anterior), 2.8 puntos porcentuales por encima del promedio nacional. 
Asimismo, Jalisco registra la misma tasa de crecimiento del promedio nacional. Sin embargo, por sexo el crecimiento de las mujeres se coloca en 0,7 puntos porcentuales superior al promedio nacional (72,722 mujeres más respecto de 2018), y los hombres experimentan un crecimiento del $0.8 \%$ ( 0.9 puntos porcentuales por debajo del promedio nacional). Sobre la base de dichos índices de crecimiento, este segmento poblacional experimenta también aumentos en su participación con respecto al total de la población: territorio nacional, $75.2 \%$ ( 0.5 puntos porcentuales mayor a 2018), Jalisco: $75.1 \%$ (0.4 puntos porcentuales por arriba de 2018), y Baja California 76.4\% (1.1 puntos porcentuales más que 2018). Por sexo, las mujeres, en el promedio nacional y en Jalisco, presentan una participación superior al 52\%, en tanto en Baja California es superior al 51\%. Por su parte, los hombres de Jalisco registran una baja en su participación de 0.4 puntos porcentuales; en Baja California la pérdida en su participación asciende a 0.8 puntos porcentuales, y el promedio nacional registra la misma participación de 2018 (cuadro 1).

\section{Cuadro 1}

Dinámica poblacional según entidad federativa y sexo

\begin{tabular}{l|r|r|r|r|r|r}
\hline & \multicolumn{3}{|c|}{ Tasa de crecimiento } & \multicolumn{3}{c}{ Variación absoluta } \\
\hline & Nacional & Jalisco & $\begin{array}{c}\text { Baja } \\
\text { California }\end{array}$ & Nacional & Jalisco & $\begin{array}{c}\text { Baja } \\
\text { California }\end{array}$ \\
\hline Población total & 1.0 & 1.0 & 1.3 & 1195748 & 84561 & 48440 \\
\hline Hombres & 1.1 & 0.9 & 0.9 & 634669 & 36256 & 15889 \\
\hline Mujeres & 0.9 & 1.2 & -1.8 & 561079 & 48305 & -32551 \\
\hline Población de 15 años y más & 1.7 & 1.6 & 2.8 & 1555134 & 95280 & 76190 \\
\hline Hombres & 1.7 & 0.8 & 1.2 & 769802 & 22558 & 15792 \\
\hline Mujeres & 1.6 & 2.3 & 4.4 & 785332 & 72722 & 60398 \\
\hline Población de menos de 15 Años & -1.1 & -3.1 & -3.1 & -359386 & -10719 & -27750 \\
\hline Hombres & -0.8 & 1.3 & 0.0 & -135133 & 13698 & 97 \\
\hline Mujeres & -1.4 & -2.4 & -6.2 & -224253 & -24417 & -27847 \\
\hline & Participación porcentual 2018 & Participación porcentual 2019 \\
\hline Población total & 0.0 & 6.6 & 2.9 & 0.0 & 6.6 & 2.9 \\
\hline Hombres & 48.3 & 48.8 & 49.2 & 48.3 & 48.8 & 49.9 \\
\hline Mujeres & 51.7 & 51.2 & 50.8 & 51.7 & 51.2 & 50.1 \\
\hline Población de 15 años y más & 74.7 & 74.7 & 75.3 & 75.2 & 75.1 & 76.4 \\
\hline Hombres & 47.5 & 48.3 & 49.7 & 47.5 & 47.9 & 48.9 \\
\hline Mujeres & 52.5 & 51.7 & 50.3 & 52.5 & 52.1 & 51.1 \\
\hline Población de menos de 15 años & 25.3 & 25.3 & 24.7 & 24.8 & 24.9 & 23.6 \\
\hline Hombres & 50.6 & 50.4 & 49.5 & 50.8 & 51.3 & 51.1 \\
\hline Mujeres & 49.4 & 49.6 & 50.5 & 49.2 & 48.7 & 48.9 \\
\hline Funte: Elo & & & & & \\
\hline
\end{tabular}

Fuente: Elaboración propia basada en INEGI, 2019. 


\section{Población económicamente activa (PEA) ${ }^{6}$}

Un primer aspecto por conocer sobre la situación de la PEA es su crecimiento anual. A escala nacional, la tasa de crecimiento fue de $2.4 \%$ (1,307,798 personas económicamente activas más), en Jalisco $2.3 \%(88,190)$ y en Baja California $2.0 \%(34,082)$. En buena medida, este ritmo de crecimiento lo explica el consistente incremento de participación de las mujeres en el mercado de trabajo, el cual, en 2019, se coloca por encima del promedio general de las tres entidades, así como también mayor que el crecimiento anual experimentado por los hombres en cada uno de los tres territorios. Por consiguiente, a escala nacional, se incorpora a la PEA un total de 908,409 mujeres; en Jalisco, 38,572, y en Baja California, 13,98. Por su parte, los hombres sumaron 399,389 a escala nacional. En Jalisco, 49,618, y en Baja California, 20,101 (cuadro 2).

Asimismo, se observa una diferenciación en los ritmos de crecimiento entre la población total y la PEA (cuadros 1 y 2), la cual se debe, solo en parte, al desfase que existe entre la población en edad de trabajar (quince años y más) y la menor de edad (menor de quince años). Sin embargo, en lo fundamental, los cambios en la PEA se ven asociados al comportamiento de los diferentes aspectos que constituyen la construcción social de la oferta y la demanda de trabajo en asociación con el modelo de desarrollo vigente (Pacheco, 1997).

Un segundo aspecto de la PEA, corresponde a la tasa de participación laboral total, masculina y femenina. Es decir, el porcentaje de la población en edad de laborar (quince años y más) que se encuentra ocupado o en búsqueda activa de trabajo. En las tres entidades territoriales, la tasa de participación laboral experimenta, en 2019, un incremento: a escala nacional representa el $61.2 \%$ (incremento de 2.4 puntos porcentuales); Jalisco, $63.0 \%$ (2.4 puntos porcentuales de incremento), y Baja California $64.4 \%$ (3.0 puntos porcentuales más que en 2018). Por su parte, la tasa de participación masculina experimenta un crecimiento en Jalisco, 78.0\% (1.0 puntos porcentuales más), así como en Baja California, $77.3 \%$ (0.6\%). Por otra parte, la participación laboral femenina experimenta un crecimiento a escala nacional de 1.2 puntos porcentuales $(44.9 \%$ ) y, en Jalisco de 0.1 puntos porcentuales (47.2\%), pero en Baja California experimenta un retroceso de 1.1 puntos porcentuales (48.5\%).

El tercer aspecto corresponde al análisis del desempeño de los dos componentes de la estructura de la PEA: uno, población ocupada; dos, población desocupada.

\subsection{Población desocupada}

En este apartado analizamos el primero de los componentes de la estructura de la PEA: el desempleo de la población que se encuentra en búsqueda activa de ocupación.

En 2019, el número de desempleados aumentó a escala nacional en un 8.4\% (se sumaron 156,336 personas); en Jalisco, aumentó 0.1\% (149 personas más). En con-

6. Se define como el universo de población en edad de trabajar, de quince años y más, que cuentan con una ocupación o se encuentran en una búsqueda activa. 
traste en Baja California se experimenta una disminución de 3.2\% respecto de 2018 (1,456 personas menos). Por sexo, el anterior comportamiento también se expresa para el caso de las mujeres: a escala nacional y en Jalisco se observa un incremento en el número de desempleadas: $12.5 \%$ (88,432 menos) y $9.3 \%$ (3,555 menos), respectivamente. En Baja California, el número de mujeres desempleadas registra un descenso del 23\% (3,636 menos). Por su parte, el número de hombres desempleados también expresa un comportamiento contrastante: a escala nacional y en Baja California registran un aumento del 5.9\% (67,904 más) y el 7.2\% (2,180 más). En Jalisco el número de hombres desempleados disminuye en .5\% (3,406 menos que en 2018).

Otro aspecto de la población desocupada corresponde a la tasa de desempleo. A escala nacional, la tasa de desempleo experimenta un incremento en 2019, en sus tres componentes: en el total, asciende al 3.5\% (0.2 puntos porcentuales más que en 2018); las mujeres registran una tasa del $3.6 \%$ ( 0.3 puntos porcentuales más), y la tasa de los hombres es del 3.5\% (0.1 puntos porcentuales más). En Jalisco, la tasa de desempleo total se mantiene en $3.0 \%$; la tasa masculina de $3.1 \%$ experimenta un descenso de 0.2 puntos porcentuales, respecto de 2018 , en tanto la tasa de desempleo femenina $(2.7 \%)$ registra un aumento de 0.1 puntos porcentuales. Por su parte, en Baja California la tasa de desempleo global $(2.7 \%)$ registra un descenso de 0.2 puntos porcentuales; la tasa masculina (3.0\%) experimenta un incremento de 0.1 puntos porcentuales, y la femenina $(1.7 \%$ ) disminuye 0.6 puntos porcentuales (cuadro 2). Esta tasa femenina es la única que se coloca por debajo del $2 \%$.

\subsection{Población ocupada}

En este subapartado analizamos el segundo de los componentes de la PEA: la población de cada una de las tres entidades territoriales que cuenta con una ocupación, así como el número total de nuevas ocupaciones.

En 2019, el número de personas que cuentan con una ocupación aumentó en el total y por sexo en las tres entidades territoriales. A escala nacional y en Baja California, el incremento fue del 2.1\%, y en Jalisco del 2.4\% (0.3 puntos porcentuales más que el promedio nacional). Dichos incrementos significan a escala nacional 1,151,462 de nuevas ocupaciones; en Jalisco, 88,041 y en Baja California, 35,538 nuevas ocupaciones. Por sexo, a escala nacional el número de mujeres, con una ocupación se incrementó en 4.0\% (819,977 más respecto de 2018); en Jalisco el incremento es del 2.4\% (35,017 más), y en Baja California el incremento ascendió al 2.6\% (13,981 más). Por su parte, la ocupación masculina registra aumentos diferenciales: a escala nacional aumentó el número de hombres en 1.0\% (331,485 más); en Jalisco, aumentó en 2.4\% (53,024 más), y en Baja California el incremento es del 1.8\%, 17,921 más (cuadro 2). En suma, la mayor dinámica en las nuevas ocupaciones la imprimen en mayor medida, en las tres entidades territoriales, las mujeres.

Cabe señalar que, no obstante que el número de ocupaciones registra un incremento en las tres entidades territoriales, solo Baja California presenta un aumento en su tasa de ocupación de 0.2 puntos porcentuales respecto de 2018 (97.5\%), en tanto 
el promedio nacional presenta un decremento de 0.1 puntos porcentuales y Jalisco un estancamiento, al situarse en el $97.0 \%$. Por sexo, solo la tasa de ocupación de hombres en Jalisco aumentó en 0.2 puntos porcentuales (96.9\%), en tanto Baja California y el promedio nacional presentan un decremento de 0.1 puntos porcentuales $(97.0 \% \mathrm{y}$ 96.5, respectivamente). Por su parte, en 2019 la tasa de ocupación de las mujeres solo aumenta en Baja California en 1.0\% (98.3\%, la mayor tasa de ocupación de las tres entidades territoriales); en Jalisco, disminuyó 0.1 puntos porcentuales (97.3\%), y el promedio nacional disminuye 0.3 puntos porcentuales (96.4\%) (cuadro 2).

En este nivel de agregación se observa que el incremento diferencial del salario mínimo en Baja California (entidad de la Zona Libre Norte) y Jalisco (entidad de la región Resto del País), así como el promedio nacional de ambas regiones, tienen un impacto positivo, toda vez que se expresa un importante incremento de la población ocupada en que destaca el correspondiente a la población femenina. En el siguiente apartado centramos el análisis en el comportamiento que registra la fuerza de trabajo asalariada en la estructura ocupacional, en razón de que el empleo asalariado constituye la población objetivo de la política de salario mínimo.

\section{Cuadro 2}

Dinámica de la PEA según sexo y tasa de participación laboral

\begin{tabular}{|c|c|c|c|c|c|c|}
\hline & \multicolumn{3}{|c|}{ Tasa de crecimiento } & \multicolumn{3}{|c|}{ Variación absoluta } \\
\hline & Nacional & Jalisco & Baja California & Nacional & Jalisco & Baja California \\
\hline PEA & 2.4 & 2.3 & 2.0 & 1307798 & 88190 & 34082 \\
\hline Hombres & 1.2 & 2.2 & 1.9 & 399389 & 49618 & 20101 \\
\hline Mujeres & 4.3 & 2.6 & 2.0 & 908409 & 38572 & 13981 \\
\hline Ocupados & 2.1 & 2.4 & 2.1 & 1151462 & 88041 & 35538 \\
\hline Hombres & 1.0 & 2.4 & 1.8 & 399389 & 53024 & 17921 \\
\hline Mujeres & 4.0 & 2.4 & 2.6 & 908409 & 35017 & 17617 \\
\hline Desocupados & 8.4 & 0.1 & -3.2 & 156336 & 149 & -1456 \\
\hline Hombres & 5.9 & -4.5 & 7.2 & 67904 & -3406 & 2180 \\
\hline \multirow[t]{3}{*}{ Mujeres } & 12.5 & 9.3 & -23.0 & 88432 & 3555 & -3636 \\
\hline & \multicolumn{3}{|c|}{ Tasa de participación laboral 2018} & \multicolumn{3}{|c|}{ Tasa de participación laboral 2019} \\
\hline & Nacional & Jalisco & Baja California & Nacional & Jalisco & Baja California \\
\hline PEA & 58.8 & 60.6 & 61.4 & 61.2 & 63.0 & 64.4 \\
\hline Hombres & 77.5 & 77.0 & 76.7 & 77.1 & 78.0 & 77.3 \\
\hline \multirow[t]{2}{*}{ Mujeres } & 43.7 & 47.1 & 49.6 & 44.9 & 47.2 & 48.5 \\
\hline & \multicolumn{3}{|c|}{ Tasa de ocupación 2018} & \multicolumn{3}{|c|}{ Tasa de ocupación 2019} \\
\hline Ocupados & 96.7 & 97.0 & 97.3 & 96.5 & 97.0 & 97.5 \\
\hline Hombres & 96.6 & 96.7 & 97.1 & 96.5 & 96.9 & 97.0 \\
\hline \multirow[t]{2}{*}{ Mujeres } & 96.7 & 97.4 & 97.7 & 96.4 & 97.3 & 98.3 \\
\hline & \multicolumn{3}{|c|}{ Tasa de desempleo 2018} & \multicolumn{3}{|c|}{ Tasa de desempleo 2019} \\
\hline Desocupados & 3.3 & 3.0 & 2.7 & 3.5 & 3.0 & 2.5 \\
\hline Hombres & 3.4 & 3.3 & 2.9 & 3.5 & 3.1 & 3.0 \\
\hline Mujeres & 3.3 & 2.6 & 2.3 & 3.6 & 2.7 & 1.7 \\
\hline
\end{tabular}

Fuente: Ela boración propia basada en INEGI, 2019. 


\section{Población ocupada según posición}

En este segundo nivel analítico de la población ocupada ${ }^{7}$ se destaca la importancia que tiene la fuerza de trabajo asalariada ${ }^{8}$ en la estructura ocupacional de cada una de las tres entidades territoriales.

\subsection{Estructura ocupacional}

Esta estructura la configuran cinco posiciones ocupacionales: empleadores, fuerza de trabajo asalariada, fuerza de trabajo no asalariada, trabajadores por cuenta propia y trabajadores no remunerados.

La fuerza de trabajo asalariada se revela como la posición ocupacional más importante en Jalisco y Baja California, así como a escala nacional. En las dos primeras entidades el número de nuevos empleos asalariados supera al número total de nuevas ocupaciones de su respectiva entidad, ${ }^{9}$ y también la tasa de crecimiento del trabajo asalariado es superior al número total de nuevas ocupaciones. Asimismo, en ambas entidades el empleo asalariado experimenta un incremento en su participación con respecto al total de la población ocupada.

En Jalisco la generación de nuevos empleos asalariados creció, en 2019, a una tasa del $4.7 \%$ (2.6 puntos porcentuales superior al promedio nacional), que en valores absolutos significa 116,357 nuevos trabajadores $(32.1 \%$, mayor al número total de nuevas ocupaciones de la entidad), los que a su vez representan el $16.1 \%$ del número total nacional de nuevos empleos asalariados en dicho año. Los hombres contribuyeron con el $67.1 \%$ de los nuevos empleos asalariados y las mujeres con el $32.9 \%$. Asimismo, la fuerza de trabajo asalariada jalisciense representa el 70.0\% (1.5 puntos porcentuales más que en 2018) respecto del número total de ocupaciones de toda la entidad (cuadros 3 y 4 ).

También en Baja California los empleos asalariados registran el mayor crecimiento, $3.2 \%$ (1.1 puntos porcentuales superior al promedio nacional), que se traduce en 37,883 nuevos trabajadores asalariados (6.6 puntos porcentuales superior al total de nuevas ocupaciones de la entidad). Estos mismos trabajadores representan el 5.2\% del total de nuevos trabajadores asalariados a escala nacional. El 57.1\% de los nuevos

7. De acuerdo con el INEGI (2019), son las "personas de 15 y más años de edad que en la semana de referencia realizaron alguna actividad económica durante al menos una hora. Incluye a los ocupados que tenían trabajo, pero no lo desempeñaron temporalmente por alguna razón, sin que por ello perdieran el vínculo laboral con este; así como a quienes ayudaron en alguna actividad económica sin recibir un sueldo o salario".

8. De acuerdo con el INEGI (2019), son solo las "personas de 15 y más años de edad que en el periodo de referencia trabajaron para un patrón o empleador del sector privado o público; y que reciben un pago, sueldo, salario o jornal".

9. A partir de este primer nivel de desagregación (estructura ocupacional según posición), el saldo positivo o negativo de nuevas ocupaciones, a escala de entidad federativa y promedio nacional, es el resultado de restar el número total de nuevas ocupaciones al número total de desaparición de ocupaciones. 
empleos asalariados bajacalifornianos corresponde a los hombres, y el $42.9 \%$ a las mujeres. De esta manera, la fuerza de trabajo asalariada representa el $72.3 \%$ del total (0.7 puntos porcentuales superior al año 2018) de población ocupada en la entidad (cuadros 3 y 4 ).

Cabe señalar que en ambas entidades el total de nuevos empleos asalariados es superior al número total de nuevas ocupaciones, en razón como señalamos, de que en este nivel de análisis prevalece la generación y desaparición de ocupaciones en alguna de las posiciones de la estructura ocupacional. Para dar cuenta de dicha situación observemos, por una parte, el comportamiento que presentan las ocupaciones por posición en Jalisco y Baja California, así como su participación porcentual en el número total de ocupaciones (estructura ocupacional).

En Jalisco, la generación de ocupaciones durante 2019, además de la fuerza de trabajo asalariada, corresponde a la de los empleadores o patrones, con 8,525 (3.8\% de crecimiento respecto de 2018), de las cuales el $82.3 \%$ de los nuevos patrones es masculino y el restante $16.7 \%$ es femenino. En contraste, las ocupaciones de fuerza de trabajo no asalariada, trabajadores por cuenta propia y trabajadores no remunerados registran en 2019 pérdidas de 13,653, 15,099 y 8,089, respectivamente. Es decir, estas tres posiciones registran una pérdida total de 36,841 ocupaciones que, sustraídas a las 124,882 nuevas ocupaciones (minuendo), generadas en las dos posiciones señaladas, restan un total de 88,041 nuevas ocupaciones (cuadro 3).

De acuerdo con lo anterior, la participación porcentual de cada una de las cinco posiciones en la estructura ocupacional tiende a reconfigurarse en 2019; como señalamos, la fuerza de trabajo representa el $70 \%$ del número total de ocupaciones (1.5 puntos porcentuales superior al registrado en 2018), los patrones el 6.2\%; (0.1 puntos porcentuales superior a 2018), la fuerza de trabajo no asalariada el $2.7 \%$ ( 0.5 puntos porcentuales inferior a 2018), los trabajadores por cuenta propia el $17.1 \%$ ( 0.8 puntos porcentuales inferior a 2018), y los trabajadores no remunerados el 3.0\% (0.3 puntos porcentuales inferior a su participación en 2018). Cabe señalar que, con excepción de los trabajadores no remunerados, en las otras cuatro posiciones ocupacionales los hombres registran una participación mayoritaria, que oscila entre el $82.3 \%$ y el $58.9 \%$ (cuadro 4).

En Baja California, como advertimos, también se generan nuevas ocupaciones. En tres posiciones se generan nuevas ocupaciones y en otras dos se registran pérdidas. El mayor número de nuevas ocupaciones corresponde a la fuerza de trabajo asalariada, con 37,883 (3.2\% de crecimiento respecto de 2018); en segundo término, se destacan las 2,672 nuevas ocupaciones de los empleadores (3.5\% de crecimiento respecto de 2018); en tercer lugar están las 2,514 nuevas ocupaciones de la fuerza de trabajo no asalariada (crecimiento del $2.7 \%$ ). En contraste se encuentran las pérdidas de ocupaciones. La mayor corresponde a los trabajadores no remunerados, con un total de 4,491 pérdidas (retroceso de $-19.0 \%$ respecto de 2018), en tanto los trabajadores por cuenta propia experimentan una pérdida de 3,040 ocupaciones $(-1.1 \%$ de retroceso respecto de 2018). Cabe señalar que, en la generación de nuevas ocupaciones y en su pérdida, los hombres y mujeres experimentan tasas de crecimiento positivas y negativas (cuadro 3). De acuerdo con lo anterior, la suma total de nuevas ocupaciones 
asciende a 35,553, diferencia entre el minuendo (43,069 nuevas ocupaciones que registran las tres posiciones señaladas) y el sustraendo (7,531 ocupaciones perdidas en las otras dos posiciones ocupacionales mencionadas).

Sobre la base de lo anterior, en 2019 con respecto al año anterior, la participación porcentual de cada una de las cinco posiciones experimenta cambios. La fuerza de trabajo asalariada representa el $72.3 \%$ del total de las ocupaciones bajacalifornianas (0.7 puntos porcentuales superior a 2018); los empleadores, representan el $4.7 \%$ (0.1 punto porcentual superior a 2018); la fuerza de trabajo no asalariada representa el $5.6 \%$ (0.1 punto porcentual superior a 2018); los trabajadores por cuenta propia representan el $16.3 \%$ ( 0.5 puntos porcentuales por debajo de su participación en 2018), y los trabajadores no remunerados, en ambos años, representan el $1.4 \%$. La participación de los hombres oscila entre el $81 \%$ (empleadores) y el $40.1 \%$ (trabajadores no remunerados); las mujeres entre el $59.9 \%$ (trabajadores no remunerados) y el $19.0 \%$ (empleadores) (cuadro 4).

Asimismo, se observa el comportamiento en la generación de nuevas ocupaciones y la pérdida de ocupaciones que presentan ambas entidades federativas, Jalisco y Baja California, con respecto al comportamiento del promedio nacional. Ambas entidades federativas presentan tasas de crecimiento superior, a escala general y por sexo, con respecto al promedio nacional de nuevas ocupaciones en la posición de fuerza de trabajo asalariada y de empleadores. En tanto Baja California presenta una tasa de crecimiento positiva en la posición de fuerza de trabajo no asalariada, Jalisco registra una tasa negativa $(-9.0 \%)$, superior en 2.4 puntos porcentuales a la tasa negativa promedio nacional. En contraste, en la posición de trabajadores por cuenta propia y no remunerados, ambas entidades presentan tasas negativas, en tanto el promedio nacional registra tasas positivas de crecimiento en general y por sexo (se destaca el crecimiento del 10\% de las mujeres en la posición por cuenta propia) (cuadro 3).

En lo que corresponde a la estructura ocupacional, el comportamiento de ambas entidades federativas con respecto al promedio nacional, el resultado es el siguiente. La participación porcentual de la fuerza de trabajo asalariada y no asalariada en el total de las ocupaciones de ambas entidades es mayor que la del promedio nacional, en lo general y por sexo, en tanto la participación de los empleadores en Jalisco (6.2\%) es superior al promedio nacional y de Baja California (cuya participación es la misma que el promedio nacional: $4.7 \%$ ). Por sexo, en ambas entidades la participación de los hombres empleadores, $82 \%$ promedio, supera la que registra a escala nacional (79.9\%), y viceversa, la participación de las mujeres en el promedio nacional supera la correspondiente a Jalisco y Baja California. En contraste, la participación porcentual de las posiciones de trabajadores por cuenta propia y no remunerada es mayor; en lo general, en el promedio nacional y por sexo se observa un comportamiento variable entre las tres entidades territoriales (cuadro 4).

De acuerdo con lo anterior, en Jalisco el incremento al salario mínimo, de 16.2\%, y en Baja California, del 100\%, produce un impacto mayormente positivo en la generación de nuevas ocupaciones que negativo en la desaparición de ocupaciones. Es decir, estos incrementos diferenciales del salario mínimo producen una mayor canti- 
dad de nuevas ocupaciones y una menor cantidad de ocupaciones pérdidas en Jalisco, Baja California y a escala nacional. Asimismo, se observa que la mayor cantidad de nuevas ocupaciones corresponde a la fuerza de trabajo asalariada, de suerte que esta posición ocupacional aumenta su participación porcentual en la estructura de Jalisco y Baja California, en tanto a escala nacional prevalece la misma participación. En suma, en 2019 la estructura ocupacional se reconfigura en Jalisco y Baja California y se mantiene a escala nacional en los términos que registra en 2018.

Además de la importancia que adquiere la fuerza de trabajo asalariada en la generación de nuevos empleos y en su participación porcentual en el número total de ocupaciones, en las tres entidades territoriales constituye el objeto de atención de la política de salario mínimo del Estado mexicano. Por lo tanto, a partir del siguiente apartado nuestro análisis se centra en dicha fuerza de trabajo, con la finalidad de establecer el impacto que produce la política de salario mínimo por sector y actividad económica, así como por tamaño de establecimiento para las tres entidades territoriales y por sexo. Es decir, en este nivel de análisis precisamos conocer las actividades económicas y el tipo de establecimientos donde se generan o desaparecen los empleos asalariados, para delimitar de mejor manera el impacto que produce la política de salario mínimo en la generación de nuevos empleos.

Cuadro 3

Estructura ocupacional: Dinámica de crecimiento según sexo

\begin{tabular}{l|c|c|r|r|r|r}
\hline & \multicolumn{3}{|c|}{ Tasa de crecimiento } & \multicolumn{3}{c}{ Variación absoluta } \\
\hline & Nacional & \multicolumn{1}{|c|}{ Jalisco } & Baja California & Nacional & Jalisco & Baja California \\
\hline Total & $\mathbf{2 . 1}$ & $\mathbf{2 . 4}$ & $\mathbf{2 . 1}$ & $\mathbf{1 1 5 1 4 6 2}$ & $\mathbf{8 8 0 4 1}$ & $\mathbf{3 5 5 3 8}$ \\
\hline Hombres & 1.0 & 2.4 & 1.8 & 331485 & 53024 & 17921 \\
\hline Mujeres & 4.0 & 2.4 & 2.6 & 819977 & 35017 & 17617 \\
\hline Empleadores & $\mathbf{0 . 8}$ & $\mathbf{3 . 8}$ & $\mathbf{3 . 5}$ & $\mathbf{2 0 0 8 6}$ & $\mathbf{8 5 2 5}$ & $\mathbf{2 6 7 2}$ \\
\hline Hombres & 0.5 & 4.0 & 2.6 & 10620 & 7267 & 1641 \\
\hline Fujeres & 1.8 & 3.2 & 7.3 & 9466 & 1258 & 1031 \\
\hline Hombres & $\mathbf{2 . 1}$ & $\mathbf{4 . 7}$ & $\mathbf{3 . 2}$ & $\mathbf{7 2 4 2 7 6}$ & $\mathbf{1 1 6 3 5 7}$ & $\mathbf{3 7 8 8 3}$ \\
\hline Mujeres & 1.9 & 5.4 & 3.0 & 398345 & 78115 & 21639 \\
\hline FT No Asalariada & 2.4 & 3.7 & 3.3 & 325931 & 38242 & 16244 \\
\hline Hombres & $\mathbf{- 6 . 6}$ & $\mathbf{- 9 . 0}$ & $\mathbf{2 . 7}$ & $\mathbf{- 1 4 1 3 5 1}$ & $\mathbf{- 1 3 6 5 3}$ & $\mathbf{2 5 1 4}$ \\
\hline Mujeres & -6.0 & -5.5 & -1.2 & -101080 & -6396 & -794 \\
\hline Por cuenta propia & -8.9 & -20.5 & 13.1 & -40271 & -7257 & 3308 \\
\hline Hombres & $\mathbf{3 . 9}$ & $\mathbf{- 2 . 3}$ & $\mathbf{- 1 . 1}$ & $\mathbf{4 6 9 7 3 9}$ & $\mathbf{- 1 5 0 9 9}$ & $\mathbf{- 3 0 4 0}$ \\
\hline Mujeres & 0.0 & -6.1 & -3.0 & 220 & -24196 & -4827 \\
\hline No remunerada & 10.0 & 3.6 & 1.5 & 469519 & 9097 & 1787 \\
\hline Hombres & $\mathbf{3 . 3}$ & $\mathbf{- 6 . 7}$ & $\mathbf{- 1 9 . 0}$ & $\mathbf{7 8 7 1 2}$ & $\mathbf{- 8 0 8 9}$ & $\mathbf{- 4 4 9 1}$ \\
\hline Mujeres & 2.3 & -4.9 & 3.5 & 23380 & -1766 & 262 \\
\hline Furata & 4.1 & -7.4 & -29.3 & 55332 & -6323 & -4753 \\
\hline
\end{tabular}

Fuente: Elaboración propia basada en INEGI, 2019. 


\section{Cuadro 4}

Estructura ocupacional: Participación porcentual según sexo

\begin{tabular}{l|r|r|r|r|r|r}
\hline \multirow{2}{*}{$\begin{array}{c}\text { Población ocupada } \\
\text { por posición }\end{array}$} & \multicolumn{2}{|c|}{ Participación Nacional } & \multicolumn{2}{c|}{ Participación Jalisco } & \multicolumn{2}{c}{ Participación Baja California } \\
\cline { 2 - 7 } & 2018 & 2019 & 2018 & 2019 & 2018 & 2019 \\
\hline Total & $\mathbf{1 0 0}$ & $\mathbf{1 0 0}$ & $\mathbf{1 0 0}$ & $\mathbf{1 0 0}$ & $\mathbf{1 0 0}$ & $\mathbf{1 0 0}$ \\
\hline Empleadores & $\mathbf{4 . 8}$ & $\mathbf{4 . 7}$ & $\mathbf{6 . 1}$ & $\mathbf{6 . 2}$ & $\mathbf{4 . 6}$ & $\mathbf{4 . 7}$ \\
\hline Hombres & 79.9 & 79.9 & 82.2 & 82.3 & 81.6 & 81.0 \\
\hline Mujeres & 20.1 & 20.1 & 17.8 & 17.7 & 18.4 & 19.0 \\
\hline FT Asalariada & $\mathbf{6 4 . 5}$ & $\mathbf{6 4 . 5}$ & $\mathbf{6 8 . 5}$ & $\mathbf{7 0 . 0}$ & $\mathbf{7 1 . 6}$ & $\mathbf{7 2 . 3}$ \\
\hline Hombres & 60.6 & 60.5 & 58.5 & 58.9 & 59.3 & 59.2 \\
\hline Mujeres & 39.4 & 39.5 & 41.5 & 41.1 & 40.7 & 40.8 \\
\hline FT No Asalariada & $\mathbf{4 . 0}$ & $\mathbf{3 . 6}$ & $\mathbf{4 . 2}$ & $\mathbf{3 . 7}$ & $\mathbf{5 . 5}$ & $\mathbf{5 . 6}$ \\
\hline Hombres & $\mathbf{7 8 . 9}$ & 79.4 & 76.8 & 79.7 & 72.9 & 70.2 \\
\hline Mujeres & 21.1 & 20.6 & 23.2 & 20.3 & 27.1 & 29.8 \\
\hline Por cuenta propia & $\mathbf{2 2 . 3}$ & $\mathbf{2 2 . 7}$ & $\mathbf{1 7 . 9}$ & $\mathbf{1 7 . 1}$ & $\mathbf{1 6 . 8}$ & $\mathbf{1 6 . 3}$ \\
\hline Hombres & 61.1 & 58.8 & 61.2 & 58.9 & 57.1 & 56.0 \\
\hline Mujeres & 38.9 & 41.2 & 38.8 & 41.1 & 42.9 & 44.0 \\
\hline No remunerada & $\mathbf{4 . 4}$ & $\mathbf{4 . 5}$ & $\mathbf{3 . 3}$ & $\mathbf{3 . 0}$ & $\mathbf{1 . 4}$ & $\mathbf{1 . 1}$ \\
\hline Hombres & 43.0 & 42.6 & 29.8 & 42.6 & 31.4 & 40.1 \\
\hline Mujeres & 57.0 & 57.4 & 70.2 & 57.4 & 68.6 & 59.9 \\
\hline
\end{tabular}

Fuente: Elaboración propia basada en INEGI, 2019.

\section{Fuerza de trabajo asalariada}

La tasa general de crecimiento promedio nacional de empleo para la fuerza de trabajo asalariada, se sitúa en $2.1 \%$ (misma tasa de crecimiento del total de la población ocupada), lo que se traduce en la generación de 724,276 nuevos empleos (de los cuales el $45.0 \%$ corresponde a las mujeres, y el restante $55.0 \%$ a los hombres). En Jalisco, la tasa general de crecimiento de la fuerza de trabajo asalariada ascendió a 4.7\% (2.6 puntos porcentuales mayor al promedio nacional), al generar 116,357 nuevos empleos, de los cuales el $32.9 \%$ corresponde a las mujeres y el $67.1 \%$ a los hombres. Para Baja California, la tasa de crecimiento del $3.2 \%$ (1.1 puntos porcentuales superior al promedio nacional) permitió generar 37,883 nuevos empleos a la fuerza de trabajo asalariada; de esta cantidad el $42.9 \%$ corresponde a las mujeres y el $57.1 \%$ a los hombres (cuadro 5).

\subsection{Por sector y actividad económica}

Se analiza el comportamiento del empleo por sector y actividad económica en razón de su tasa de crecimiento y de los saldos que producen los encuentros (generación de 
empleo) y los desencuentros (desempleo) entre la oferta y la demanda de la fuerza de trabajo asalariada. Es decir, los encuentros y desencuentros entre mano de obra y empleadores.

\subsubsection{Por sector económico}

En primer lugar, se observa que la tasa de crecimiento promedio nacional de la fuerza de trabajo asalariada del sector primario fue del $3.4 \%$, lo que se traduce en la generación de 94,594 nuevos empleos de los cuales el $13.0 \%$ es femenino y el $87.0 \%$ es masculino. En Jalisco, la fuerza de trabajo asalariada del sector primario registra un descenso de $-2.4 \%$, lo que significa que se perdió un total de 4,890 empleos, de los cuales el $99.2 \%$ corresponde a los hombres, y las mujeres solo perdieron el $0.8 \%$ de los empleos . En Baja California, el empleo del sector primario creció a una tasa del $6.7 \%$ (3.3 puntos porcentuales superior al promedio nacional), lo que representa 4,557 nuevos empleos, de los cuales el $18.2 \%$ corresponde a las mujeres y el restante $81.8 \%$ a los hombres (cuadro 5).

En segundo lugar, en el sector secundario el crecimiento promedio nacional del empleo asalariado fue del $0.9 \%$, es decir, se generaron 91,399 nuevos empleos los cuales fueron ocupados en un $61.7 \%$ por hombres y el restante $38.3 \%$ por mujeres. En Jalisco, el crecimiento del empleo asalariado fue del 8.3\% (7.4 puntos porcentuales superior al promedio nacional), 60,135 más trabajadores contratados, de los cuales el $11.6 \%$ corresponde a las mujeres y el $88.4 \%$ a los hombres. En Baja California el empleo asalariado en el sector secundario creció en el 3.5\% (2.6 puntos porcentuales superior al promedio nacional), al generar 15,950 nuevos empleos: el 18.1\% lo ocupan las mujeres y el restante $81.9 \%$ los hombres (cuadro 5).

En tercer lugar tenemos al sector terciario. El crecimiento promedio nacional de dicho sector fue del 2.5\% (0.4 puntos porcentuales superior al crecimiento de la fuerza de trabajo asalariada), lo que representa 535,454 nuevos empleos (superior en $82.3 \%$ al generado en el volumen total del sector primario, y en el $82.9 \%$ también superior al volumen generado en el sector secundario). Del número total de nuevos empleos, el 50.7\% los ocupan las mujeres, y el restante $49.3 \%$ los hombres. En Jalisco, el empleo asalariado del sector terciario creció en 3.4\% (4.9 puntos porcentuales inferior a la tasa del sector secundario), al generar 53,620 nuevos empleos ( $7.2 \%$ inferior al volumen del sector secundario), de los cuales el $54.3 \%$ es ocupado por las mujeres y el $45.7 \%$ por hombres. En Baja California aumenta el empleo asalariado en el $2.9 \%$ (3.8 puntos porcentuales inferior al crecimiento de su sector primario y 0.6 puntos porcentuales inferior al de su sector secundario). Este crecimiento equivale a un volumen de 19,381 nuevos empleos (76.5\% por arriba del crecimiento de su sector primario y $17.7 \%$ superior a su sector secundario), de los cuales el $69.0 \%$ corresponde a las mujeres y el $31.0 \%$ a los hombres (cuadro 5). Como se observa, en las tres entidades territoriales el mayor número de empleos asalariados generados en 2019 corresponde al sector terciario, empleos que, a su vez, en mayor número, fueron asignados a las mujeres. 


\subsubsection{Comportamiento del empleo asalariado por actividad económica no agropecuaria: construcción, manufactura, comercio y servicios}

En el espectro nacional la industria de la construcción registra un decremento del $-2.5 \%$ interanual, lo que significa una pérdida de 74,916 empleos, o de desencuentros entre la oferta y la demanda de fuerza de trabajo; el $72.8 \%$ corresponde a los desencuentros que enfrentó la fuerza de trabajo asalariada masculina, y el restante $27.2 \%$ a desencuentros femeninos. En contraste, en Jalisco el crecimiento del empleo en esta industria es del 4.2\%, lo que significa una creación de 8,391 nuevos empleos, resultado de una pérdida de 767 empleos femeninos y de la creación de 9,158 empleos masculinos. En Baja California, dicha industria también registra un decremento de $-8.5 \%$ (6.0 puntos porcentuales superior al decremento nacional), lo cual significa una pérdida de 6,464 empleos: el $85.6 \%$ corresponde a los hombres y el14.4\% a las mujeres (cuadro 5).

Por su parte, la industria manufacturera experimenta en el ámbito nacional un crecimiento del $2.9 \%$, lo que favorece la generación de 194,432 nuevos empleos, de los cuales el $73.5 \%$ fue otorgado a los hombres y el $26.5 \%$ a las mujeres. La actividad manufacturera en Jalisco aumentó el número de nuevos empleos asalariados en $10.7 \%$ (6.0 puntos porcentuales superior a la tasa de crecimiento del total de empleo asalariado jalisciense); este crecimiento representa la creación de 55,209 nuevos empleos: $84.8 \%$ masculinos por $15.2 \%$ femeninos. En Baja California el crecimiento fue de $5.3 \%$ (2.1 puntos porcentuales superior a la tasa de crecimiento del total de empleo asalariado de dicha entidad), lo que, en volumen, representa 19,515 nuevos empleos: el $90.8 \%$ otorgado a hombres y el $9.2 \%$ a mujeres (cuadro 5).

En lo correspondiente al comercio, la tasa de crecimiento nacional fue del $4.0 \%$ (1.9 puntos porcentuales superior a su tasa general de trabajo asalariado), lo que se traduce en la creación de 201,311 nuevos empleos: el 51.5\% corresponde a las mujeres y el $47.5 \%$ a los hombres. En Jalisco, el empleo asalariado en el comercio creció en el $5.6 \%$ ( 0.9 puntos porcentuales superior a la tasa de crecimiento del empleo asalariado total de la entidad), lo que permite generar 22,693 nuevos empleos: $87.0 \%$ para mujeres y $13.0 \%$ para hombres. También en Baja California, el comercio registra un crecimiento del $2.9 \%$ ( 0.3 puntos porcentuales inferior a su tasa de crecimiento general de nuevos asalariados), el cual contribuye con 5,513 nuevos empleos: el 96.8\% obtenido por hombres y el $3.2 \%$ por mujeres (cuadro 5).

Por último, se encuentra la tasa de crecimiento en los servicios. El crecimiento promedio a escala nacional fue del $2.0 \%$, lo que permitió crear 334,143 nuevos empleos (el mayor número de nuevos empleos del conjunto de actividades económicas principales): el 50.3\% de los nuevos empleos corresponde a los hombres y el $49.7 \%$ a las mujeres. En Jalisco, el empleo asalariado en los servicios creció a una tasa del $2.7 \%$, la que permite contribuir con 30,927 nuevos empleos (el segundo mayor número de nuevos empleos generados en la entidad por actividad principal), resultado de un incremento de 35,931 nuevos empleos femeninos y pérdida de 5,004 empleos masculinos. En Baja California, la tasa de crecimiento asciende al 2.9\%, la cual se traduce 
en 13,868 nuevos empleos (segunda posición en la creación de empleo de la entidad): $95.1 \%$ para las mujeres y $4.9 \%$ para los hombres (cuadro 5).

\section{Cuadro 5}

Dinámica de crecimiento de nuevos empleos asalariados según sector y actividad económica

\begin{tabular}{l|c|c|c|c|c|c}
\hline \multirow{2}{*}{$\begin{array}{l}\text { Sectory Actividad } \\
\text { Económica }\end{array}$} & \multicolumn{3}{|c|}{ Tasa de crecimiento } & \multicolumn{3}{c}{ Variación absoluta } \\
\cline { 2 - 7 } & Nacional & Jalisco & Baja California & Nacional & Jalisco & Baja California \\
\hline Total de asalariados & 2.1 & 4.7 & 3.2 & 724276 & 116357 & 37883 \\
\hline Primario & 3.4 & -2.4 & 6.7 & 94594 & -4890 & 4557 \\
\hline Secundario & 0.9 & 8.3 & 3.5 & 91399 & 60135 & 15950 \\
\hline Otros & -7.0 & -25.6 & 49.4 & -28117 & -3465 & 2899 \\
\hline Construcción & -2.5 & 4.2 & -8.5 & -74916 & 8391 & -6464 \\
\hline Manufacturas & 2.9 & 10.7 & 5.3 & 194432 & 55209 & 19515 \\
\hline Terciario & 2.5 & 3.4 & 2.9 & 535454 & 53620 & 19381 \\
\hline Comercio & 4.0 & 5.6 & 2.9 & 201311 & 22693 & 5513 \\
\hline Servicios & 2.0 & 2.7 & 2.9 & 334143 & 30927 & 13868 \\
\hline No especificado & 2.3 & 296.0 & -21.4 & 2829 & 7492 & -2005 \\
\hline
\end{tabular}

Fuente: Elaboración propia basada en INEGI, 2019.

\subsection{Tamaño de establecimiento}

Se observa que el mayor número de nuevos empleos asalariados, en las tres entidades territoriales, es generado por los establecimientos no agropecuarios. Estos nuevos empleos asalariados es el resultado de los encuentros y desencuentros entre fuerza de trabajo y empleadores.

En Jalisco, los establecimientos no agropecuarios son los únicos que generan nuevos empleos asalariados (128,519, los cuales representan el $23.8 \%$ del total nacional), a una tasa de crecimiento del $5.7 \%$. Este total de nuevos empleos asalariados es el resultado de 194,813 nuevos encuentros y 66,294 desencuentros. Entre los encuentros entre fuerza de trabajo y empleadores se destacan, en primer lugar, los 77,196 nuevos empleos generados por las grandes empresas $(39.6 \%$ respecto del número total de encuentros).

En segundo lugar se encuentran los 51,004 nuevos empleos (con una tasa de crecimiento del $9.1 \%$ respecto de 2018) generados por las microempresas. Cabe señalar que de esta última cantidad de nuevos empleos el $70.2 \%$ fue generado por microempresas que no cuentan con un establecimiento (los cuales son considerados como precarios o informales), el resto de nuevos empleos asalariados $(15,180)$ lo generan microempresas con establecimientos. En tercer lugar se destacan las medianas empresas, las cuales generan 36,180 nuevos empleos asalariados (18.6\% respecto del total de nuevos encuentros). En cuarto lugar se encuentra otro tipo de establecimientos, con 
una generación de 30,433 nuevos empleos ( $15.6 \%$ respecto del número total de nuevos encuentros), con una tasa de crecimiento del $20.5 \%$ con respecto a 2018 (la de mayor dinamismo del todo el conjunto de establecimientos no agropecuarios). En contraste se encuentran los establecimientos donde se generan desencuentros entre la fuerza de trabajo y los empleadores. En primer término aparecen las pequeñas empresas, con una pérdida de 52,384 empleos asalariados (el 79.0\% del total de desencuentros), las cuales representan un retroceso de $-6.9 \%$ respecto de 2018 . En segundo término se encuentra la pérdida de 13,910 empleos generados en el sector público (tres niveles de gobierno), los cuales significan un retroceso de $-11.5 \%$ respecto de 2018 . Cabe señalar que, de acuerdo con la tasa de crecimiento que presenta la mayoría de establecimientos jaliscienses, se observa una mayor dinámica de crecimiento respecto del promedio nacional en la generación de nuevos empleos (cuadro 6).

En Baja California los establecimientos no agropecuarios presentan un saldo a favor de nuevos empleos asalariados. En primer lugar, el rubro de establecimientos no especificados sesga y limita el análisis de la información estadística, toda vez que 24,713 asalariados no especificaron el tamaño de establecimiento donde laboran, lo que altera el resultado, principalmente, de las microempresas que generan nuevos empleos asalariados. No obstante lo anterior, podemos destacar que las grandes empresas (maquiladoras) generan el mayor número de nuevos empleos $(24,975)$, con una tasa de crecimiento del 8.2\%. En segundo lugar sobresalen los 4,085 nuevos empleos generados por las pequeñas empresas (a diferencia de Jalisco y a escala nacional, donde se registran desencuentros o pérdidas de empleo). En tercer lugar se destacan los 4,044 nuevos empleos generados por el sector público. En contraste, las medianas empresas registran una pérdida de 22,236 empleos, así como en otro tipo de establecimientos se perdieron 2,276 empleos asalariados (cuadro 6).

\section{Cuadro 6}

Dinámica de crecimiento según tamaño de establecimiento

\begin{tabular}{l|r|r|r|r|r|r}
\hline \multirow{2}{*}{ Establecimientos } & \multicolumn{3}{|c|}{ Tasa de crecimiento } & \multicolumn{3}{c}{ Variación absoluta } \\
\cline { 2 - 7 } & Nacional & Jalisco & Baja California & Nacional & \multicolumn{1}{c}{ Jalisco } & Baja California \\
\hline Total de asalariados & 2.1 & 4.7 & 3.2 & 724276 & 116357 & 37883 \\
\hline Agropecuario & 3.4 & -2.4 & 6.7 & 94594 & -4890 & 4557 \\
\hline No agropecuarios & 1.8 & 5.7 & 0.8 & 540737 & 128519 & 8613 \\
\hline Microempresa & 3.9 & 9.1 & 0.2 & 297080 & 51004 & 417 \\
\hline Sin establecimiento & 1.9 & 22.0 & -2.2 & 53085 & 35824 & -1430 \\
\hline Con establecimiento & 5.1 & 3.8 & 1.4 & 243995 & 15180 & 1847 \\
\hline Pequeña empresa & -1.2 & -6.9 & 1.4 & -94603 & -52384 & 4085 \\
\hline Mediana empresa & -0.9 & 7.9 & -11.3 & -49184 & 36180 & -22236 \\
\hline Gran empresa & 3.9 & 35.3 & 8.2 & 187334 & 77196 & 24975 \\
\hline Gobierno & 4.0 & -11.5 & 8.4 & 88242 & -13910 & 4044 \\
\hline Otros & 4.8 & 20.5 & -6.0 & 111868 & 30433 & -2672 \\
\hline No especificado & 4.3 & -32.8 & 46.7 & 88945 & -7272 & 24713 \\
\hline
\end{tabular}

Fuente: Elaboración propia basada en INEGI, 2019. 
De acuerdo con lo anterior, si bien impera el mayor número de nuevos empleos asalariados sobre el menor número de desaparición de dichos empleos, los impactos siempre se revelan como positivos y negativos en las tres entidades territoriales y según sexo.

\section{Consideraciones finales}

El incremento al salario mínimo en 2019, del 16.2\% en la región resto de México, así como de $100 \%$ en la región Zona Libre de la Frontera Norte de México, tuvo impactos mayormente positivos en la generación de nuevos empleos asalariados, y también dicho incremento produjo, en menor número, impactos negativos o desaparición de empleos asalariados en las tres entidades territoriales analizadas: Jalisco, Baja California y promedio nacional, así como en los tres niveles analíticos (estructura ocupacional, fuerza de trabajo por sector y actividad económica y tamaño de establecimiento) y en la fuerza de trabajo femenina y masculina.

Cuatro son los matices particulares que signan el aumento de nuevos empleos en el periodo de análisis: uno, la fuerza de trabajo asalariada incrementa su participación porcentual en la estructura ocupacional de Jalisco y Baja California y a escala nacional se mantiene. Por sector económico el mayor número de empleos en Baja California y a escala nacional los genera el terciario, y en Jalisco los genera el sector secundario. Las pérdidas de empleo asalariado solo ocurren en el sector primario de Jalisco; dos, por actividad económica, en Jalisco, Baja California y a escala nacional las manufacturas generan el mayor número de nuevos empleos asalariados; tres, en contraste, las mayores pérdidas de empleo en Jalisco las registran las actividades agropecuarias y forestales; en Baja California y a escala nacional la mayor pérdida de empleo ocurre en la industria de la construcción; cuatro, por tamaño de establecimiento, la mayor generación de empleos en Jalisco y Baja California correspondió a la gran empresa, y a escala nacional la microempresa. Las pérdidas en Jalisco y a escala nacional ocurren en las pequeñas empresas, y en Baja California en las medianas empresas. En términos de sexo, el mayor número de nuevos empleos fue cubierto por hombres, pero por sector en el primario y secundario la mayoría corresponde a los hombres y en el terciario la mayoría de nuevos empleos es ocupada por mujeres. Por actividad económica, en manufacturas, comercio y agropecuarios la mayoría de nuevos empleos asalariados es masculina y solo en servicios en general la mayoría es femenina. En suma, los impactos positivos (creación de nuevos empleos) del incremento del salario mínimo son diferenciales por entidad territorial, sector y actividad económica, así como entre sexos.

La prevalencia de nuevos empleos para la fuerza de trabajo asalariada confirma, al igual que lo sustentan los estudios realizados en países desarrollados y en desarrollo, que el incremento sustancial al salario mínimo no constituye un impedimento para la generación de dichos empleos. Cabe señalar que, incluso, el incremento de nuevos empleos asalariados ocurre en un entorno de muy débil crecimiento de la actividad económica a escala nacional (0.3\% en el segundo trimestre de 2019); Jalisco 
y Baja California (de acuerdo con el Indicador Trimestral de la Actividad Económica Estatal) experimentan un crecimiento del $2.3 \%$ y del $1.1 \%$. Asimismo, en el segundo trimestre de 2019 la inversión extranjera directa (IED), experimenta a escala nacional y en Baja California una caída de $-18.7 \%$ y de $-17.4 \%$, respectivamente. En contraste la IED en Jalisco experimenta un crecimiento del 9.4\% (Banco de México, 2019). Cabe señalar que las tasas de crecimiento del empleo asalariado, en Jalisco y Baja California, resultan superiores a las tasas de crecimiento de los índices macroeconómicos señalados con antelación.

\section{Referencias}

Banco de México (2019). Disponible en: https://www.banxico.org.mx/. Consultado: 17 de septiembre de 2019.

Campos Vázquez, R. M.; Esquivel, G. y A. S. Santillán Hernández (2017). "El impacto del salario mínimo en los ingresos y el empleo en México". Revista de la CEPAL, núm. 122, agosto.

Card, D. y A. Krueger (1994), "Minimum Wages and Employment: A Case Study of the Fast-Food Industry in New Jersey and Pennsylvania". American Economic Review 84(4), pp. 772-793.

Diario Oficial de la Federación (DOF) (2018). Disponible en: https://www.dof.gob.mx/ index.php?year $=2018 \&$ month $=12 \&$ day $=26$. Consultado: 22 de agosto de 2019.

Dube, A. W. L. y M. Reich (2010), "Minimum Wage Effects across State Borders: Estimates using Contiguous Counties". The Review of Economics and Statistics, 92(4), pp. 945-964.

Garza Toledo, E. de la (2000). "La construcción socioeconómica del mercado de trabajo y la reestructuración productiva en México". En Enrique de la Garza Toledo (Coord.). Reestructuración productiva, mercado de trabajo y sindicatos en América Latina. Consejo Latinoamericano de Ciencias Sociales Editorial (CLACSO).

Gindling, T. H. y K. Terrell (2010). "Minimum Wages, Globalization, and Poverty in Honduras". World Development, Elsevier, vol. 38(6), pp. 908-918, junio.

INEGI (2019a). Encuesta Nacional de la Ocupación y el Empleo (ENOE). Disponible en: https://www.inegi.org.mx/enoe. Consultado: 14 de septiembre de 2019.

- (2019b). Indicador trimestral de la actividad económica estatal. Disponible en: https://www.inegi.org.m/temas/itaee/. Consultado: 12 de septiembre de 2019.

Kaplan, D. S. y F. Pérez Arce Novaro (2006). El efecto de los salarios mínimos en los ingresos laborales de México. El Trimestre Económico 73(289), pp. 139-173.

Lemos, S. (2009). "Minimum Wage Effects in a Developing Country". Labor Economics, vol. 16, núm. 2, pp. 224-237.

Neumark, D. y W. L. Wascher (2008). Minimum Wages. Cambridge, MA: MIT Press.

Pacheco, E. (1997). "Cambios en la población económicamente activa: 1900-1995". DemoS, núm. 30. México, DF. 
Stewart, M. (2003). "The Employment Effects of the National Minimum Wage". University of Warwick. Disponible en: http://www2.warwick.ac.uk/fac/soc/economics/ staff/academic/stewart/wp/mwsum.pdf. Consultado: 22 de octubre de 2019. 